\title{
Model-based simulation of driver expectation in mountainous road using various control strategies
}

\author{
Wuhong Wang, Weiwei Guo*, Yan Mao \\ School of Mechanical Engineering, Beijing Institute of Technology, Beijing 100081, China
}

\author{
Xiaobei Jiang ${ }^{1,2}$ \\ 1Department of Transportation Engineering, Beijing Institute of Technology, Beijing 100081, China \\ 2Lehrstuhl für Ergonomie, Technische Universität München, München 85747, Germany \\ Hongwei GUO \\ School of Mechanical Engineering, Beijing Institute of Technology, Beijing 100081, China \\ Geert Wets
}

Transportation Research Institute, Hasselt University, Wetenschapspark 5 bus 6, 3590 Diepenbeek, Belgium

Weihan Zhang

Research Institute of Highway Ministry of Transport, Beijing, 100088

Received 20 July 2011

Accepted 25 November 2011

\begin{abstract}
Driver expectation is about driver's objective response and expectation for various traffic environments. It might directly influence the driving safety and traffic operation. This paper discusses in enough detail the shaping mechanism of driver expectation while considers road geometer parameters, driver behavior and vehicle performance in mountainous road. Combined various geometer parameters with different driving characteristics, the vehicle trajectory and speed were selected as key factors for describing completely the vehicle's operation condition. And then, the desired trajectory models were established using six basic forms of the desired trajectory. Based on the vehicle dynamics and control strategy, a desired speed model that consisted of the desired trajectory, the driver's experience and vehicle parameter was developed, the numerical simulation results show the simulation-based model of driver expectation is used to driving behavior analysis in mountainous road.
\end{abstract}

Keywords: Control strategies, Driver expectation, Desired trajectory, Numerical simulation.

\section{Introduction}

Driver expectation is about driver's objective response and expectation for various traffic environments. It is the internal cause of driving decision-making and operation. A great many researchers attached great importance to the function of the driver expectation in the process of driving and the effect of traffic safety. The road traffic environment should correspond with driver expectation, which is significant concept generally accepted by the developed counties. While road conditions and driver expectation have observably difference, it will result in the incoordination between

\footnotetext{
* Corresponding author: guoweiwei01@bit.edu.cn
} 
human and road, and then cause the growth of the driver reaction time and errors, as a result, it will increase risk of traffic accidents. As far back as the 1970s the United States, some studies about design consistency problems related driver expectation has been started, until 90s the Self-explaining roads(SER) was proposed. The report ${ }^{1}$ points out the driver expectation that effect all aspects of highway design and operation, speed and accuracy of traffic information processing and expression of information. In the 2007 DVM simulates the vehicle's steering, braking and acceleration processes through the models of the driver's perception, cognition, control behavior and so on to evaluate the speed and the trajectory of the vehicle ${ }^{1}$. These studies reflect the concept which road design should correspond with the driver expectation respectively, but less for the directed driving expectation and reversely, more for the related research (driver behavior model, driver workload). In these years especially with People-oriented idea deeply rounted, human behavior and psychological activity in the process of driving have become the hot topic in the field of transportation engineering. These researches are an important foundation of driver expectation and desires based on driving safety analysis ${ }^{2}$.

From 1938 Gibson and Crooks proposed the field-analysis theory of vehicle travel to now, as many as 20 kinds of driving behavior theoretical models were put forward by scholars from various countries. Since the last century middles period, the development of driver behavior characteristics model has roughly experienced Compensation Follow Theory, Preview Follower Theory and Direction and Speed Integrated Control Theory ${ }^{3,4}$. Compensation Follow Theory was firstly proposed by America scholar McRuer, but Preview Follower Theory was firstly proposed by Japanese scholar Kondo in Linear prediction model in 1968. From 80s, the model that researcher proposed almost the same are Preview Follower Theory ${ }^{5}$. More recently, Wang went deeply into discuss the theory and method of driving behavior ${ }^{6,7}$. Jan Tornros tested the driver brain load under the real road conditions (rural roads, urban roads, complex traffic environment, and simple driving environment) through experiment. He proposed complexity of the external environment directly related to the driver brain load greatly and ranked its' effect degree. In 2002, Dario used of the ACT-R to predict and model driver behavior at
Drexel University. He regard the process of driving as multi-task process, and driver behavior is divided into three parts: perception information of front and rear road, making decisions, finding control variable to operate vehicle safety driving.

About studies on psychology and physiology, Torsvall , Akerstedt and Kecklund maked use of Electroencephalogram(EEG) to analyse relationship between doze and accident during the driving. Chung, Korea, used EEG to study the state of the driving in different road geometer, and proposed that the length of straight line of road can maintain an appropriate awakening level of the driver is about $4.2 \mathrm{~km}$. Yanchuan, Furuya Shirofrom Japan started to use heart rate, blood pressure and other physiological indicators to study the relationship between forest road geometer indicators and driving psychological, physiological response. Furuiehi, Tomok, Iwasak and Masat, from Japan, in about 2002, measured the vehicle's speed, eye movement and heart rate and other parameters in order to study different problems in traffic accidents caused by two equivalent geometer parameters "S"-shaped road traffic accidents, analyzed results of test of these two roads.

In conclusion, the research of driver expectation, the psychology, and the physiological and related driving behavior mainly focuses on the field of traffic safety and vehicle engineering. The object of the research of field in vehicle engineering is evaluating the operation stability of the vehicle, which consider mainly of vehicle performance conditions, and take roadway geometrics only as a secondary conditions, and traffic safety field pay more attention to the safety of the road design and scientific. The DVM of United States is mainly for the evaluation of simulation system and the model is based on the foreign traffic conditions, the research involves lots of parameters calibration. The roadway geometrics in mountainous area of our country is complex, the drivers' operation environment and vehicle characteristics are different from abroad, and DVM's domestic application has limitations. To study domestic drivers' desire drive thoroughly and further, the road linear characteristics and vehicles characteristics as well as drive psychological need to be analyzed as equal factors. Therefore this paper taken by the phenomenon to the ideas of the nature, firstly the roadway geometrics and driving condition in mountainous area of our country is observed, and the 
parameters which can respond the running trajectory and speed are selected, the mechanism of the mountainous highway driver expectation is studied further, and then a corresponding driver expectation model is set up, finally, the results are compared and analyzed with actual observation.

\section{Driver expectation shaping mechanism}

The desire produced follows person's entire mechanism to produce, and is affected and by the experience, ability, personality, needs, interests and other individual psychological factors, as well as driving experience, traffic level of awareness, safety attitudes. Driver expectation is mainly affected by the transportation environment, the vehicles characteristic and the drivers' characteristic these three key aspect influences. In view of the research mountainous area road environment, according to the curve characteristic and path condition which is observes, before the drivers enter the plane curve, they will make an estimate to the future vehicle trajectory, which is the desired trajectory. Meanwhile, it is estimated that by the beginning of the curve, speed, steering wheel rotation rate. Therefore, driver expectation will to be researched in the driver's desired trajectory and hopes speed, the relationship between the two parts are shown in Fig.1.

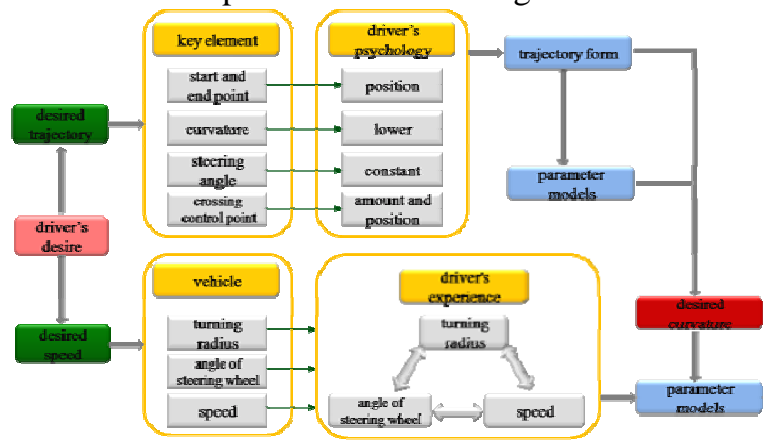

Fig.1 Form principle of driver expectation

The starting point of the curve depends on the driver's desire path form, the driver will estimate the desired trajectory according to the driving experience of the similar curve formerly had gone, and then decided to move the starting point. Speed and the steering wheel rotation range of experience told the driver and combined with the actual state of the road traffic in decision-making. Experience of the pilot of the performance of the vehicle is familiar with the process, through constant practice of moving an amendment to the repeated. To determine traffic, the radius, the speed and the steering wheel turned in a relationship exists between. The driver to produce the desired by the through a period of driving, with the traffic to performance, the radius, the speed and the steering wheel rotation in the underlying rule and desire results by the curvature of the steering wheel turned and the speed at which the amplitude.

\section{Driver's desired trajectory}

In certain road conditions, desired trajectory can be defined as desire of vehicle's running trajectory of future time by the processing and analyzing of thinking and the perception of ahead linear path with a certain range, when drivers were in the desired and natural environment. Desired trajectory changed the manipulation of vehicles. By turning, accelerating and braking the desire of driving trajectory realized. Then vehicles' actual trajectory will close to or overlap the desired trajectory. However, due to the accuracy of operation or the deviation of desired trajectory, actual trajectory would have big difference with desired trajectory. As shown in the Fig.2, when the vehicle drives to a position before the curve of the road, drivers will have a desire of future trajectory. However different drivers have different tendencies. Some drivers may desire to cross the curve along the central line of driveway, as dotted line B shows. And some drivers may desire to cross the curve by closing to the outside of driveway and even occupying the opposite driveway, as dotted line $\mathrm{A}$ and $\mathrm{C}$ shows.

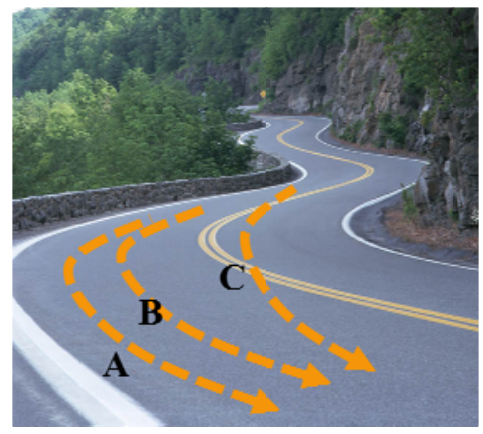

Fig.2 Illustration of desired trajectory

\subsection{Disired trajectory of geometric limit}

For different driving habits and road linear features, desired trajectories are different, but the range of road space and vehicle performance have an objective limit on 
the desired trajectory, and the driver's subjective expectations are limited by the law and behave in certain, and this is also the theoretical premise on the existence of desired trajectory. In order to facilitate the entirety rules, some limitations are listed as follows.

1) The drivers desire a constant speed to go through the circular curve, the vertical acceleration is 0 .

2) The drivers desire a constant lateral acceleration to go through the circular curve.

3) The drivers desire a smaller curvature to go through the circle curve.

4) The driver's desired trajectory's steering angle and the road circle's steering angle are the same.

5) The drivers desired trajectory's cornering which relative to the midline requires to meet the maximum limit.

6) We suppose the transition curve approximated as the circular curve, and the radius is the same with the circular curve.

From the desired trajectory's meaning and the above limits, the driver's desired trajectory for the horizontal circular curve is also the circular curve. As shown below, from the perspective of analytic geometry, circular curve endpoints, curvature, central angle is the basic variables to determine the parameter equation, and it is corresponded to the basic control points, such as the desired trajectory's starting point, ending point, curvature, steering angle and so on. In addition to these basic control points, the location relationship between the desired trajectory and the real road determined its main features. Circular curve to the real road, the lane centerline, the road centerline regarded as the basic point to the relationship between the desired trajectory and the actual road. Referred to the below coordinate system, the longitudinal offset of the desire trajectory endpoint includes ahead, behind, parallel the three cases relative to the real road curve, the lateral offset of the desired trajectory endpoint includes inside offset, outside offset and non offset the three cases relative to the real road centerline. The Intersection control points between the desired trajectory and the lane centerline include $0,1,2$ three cases. In addition, when some of the vehicles go through the horizontal circular curve, they will occupy the opposite lane to shift, we use the intersection control points to between the desired trajectory and the central line of separation to describe this situation, and we regard it as a special case.

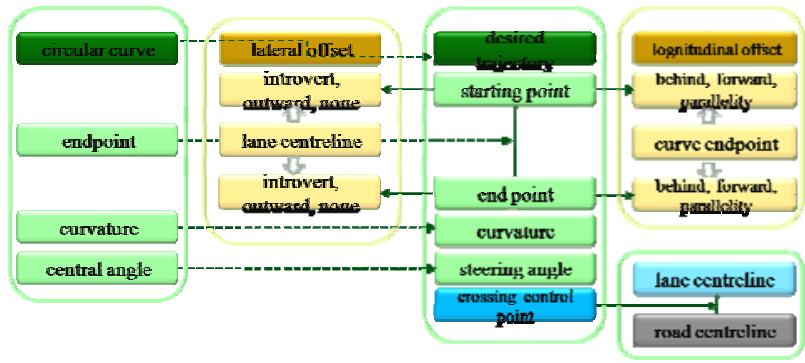

Fig.3 The analysis of the critical control points

\subsection{The basic form of desired trajectory}

Drivers desire to reduce the curvature, steering angle keeping constant, combined the above analysis, for the concave curve (right shift) we conclude the following six basic form of desired trajectory. As the Fig.4 9 shows, the blue line is the desired trajectory.

(1) Drivers want to drive along the lane outside the center line, there are no intersection with the center line, desired trajectory's starting point is advanced, and the ending point is behind. And the curvature is reduced.

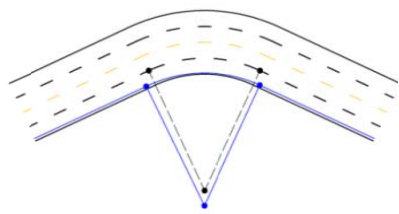

Fig.4 Concave curve of the driver's desired trajectory (a)

(2) Drivers desire to drive along the lane inside

the center line when they derived to the curve, and desire to drive the lane outside the center line at the center of the curve, and desire to drive the lane inside the center line when they leave the curve, there are two intersection with the center line of the lane. Desired trajectory's starting point is advanced, and the ending point is left behind. And the curvature is reduced.

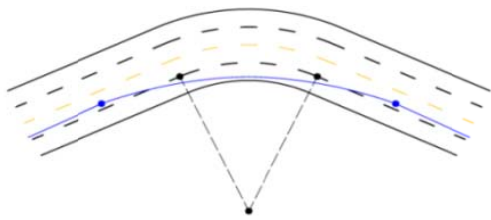

Fig.5 Concave curve of the driver's desired trajectory (b)

(3) Drivers desire to drive along the lane inside the center line when they derived to the curve, and desire to drive the lane outside the center line when they leave the curve, there is a interaction between the uncertain position of the middle curve and the center line of the lane. Desired trajectory's starting point is advanced, and the ending 
point is left behind. And the curvature is reduced.

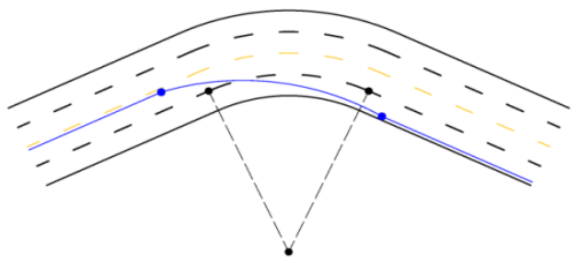

Fig.6 Concave curve of the driver's desired trajectory (c)

(4) Drivers desire to drive along the lane outside the center line when they derived to the curve, and desire to drive the lane inside the center line when they leave the curve, there is a interaction between the uncertain position of the middle curve and the center line of the lane. Desired trajectory's starting point is advanced, and the ending point is left behind. And the curvature is reduced.

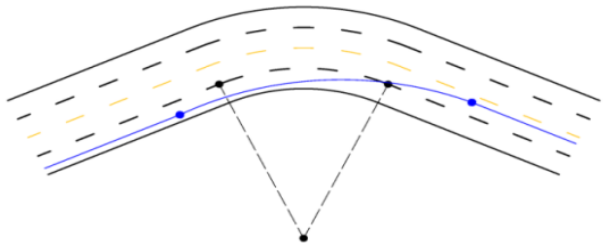

Fig.7 Concave curve of the driver's desired trajectory (d)

(5) The driver always desire to drive along the middle line of the lane, the ending point of the desired trajectory is the same with the natural curve. And the curvature of the desire trajectory is still the same.

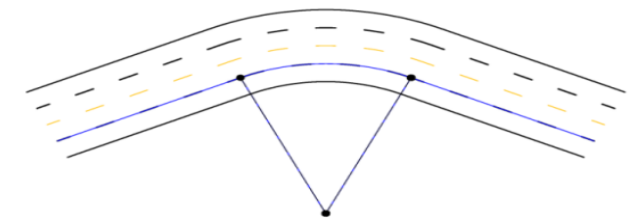

Fig.8 Concave curve of the driver's desired trajectory (e)

(6) Drivers desire to drive along the lane inside the center line. Starting point is left behind, and the ending point is advanced. And the curvature is reduced.

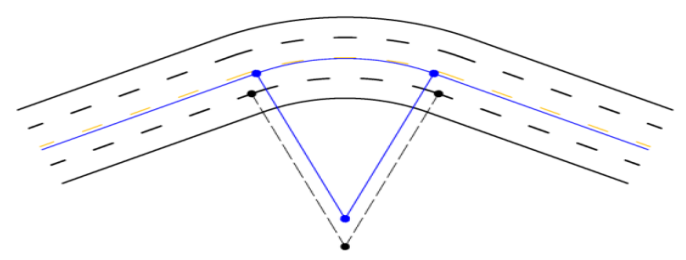

Fig.9 Concave curve of the driver’s desired trajectory (f)

\subsection{Parameter models}

The coordinate system needed to establish in order to describe vehicles, road geometer parameter and trajectory easily. As shown in Fig.10, make vehicle location to the origin of coordinates at time $t_{0}$, the $\boldsymbol{X}$-axle of the coordinates is perpendicular to the straightway road, the $\boldsymbol{Y}$ -axle of the coordinates is parallel with the straightway road, the $Z$-axle of the coordinates is perpendicular to $\boldsymbol{X Y}$-plane, the centre of a circle curve is point $\boldsymbol{C}$, the coordinate value of the centre of a circle curve is $\left(\boldsymbol{X}_{\boldsymbol{C}}, \boldsymbol{Y}_{\mathrm{C}}\right)$,the radius of curve is $R$, the steering angle of curve is $\theta$,the distance between the origin $\boldsymbol{O}$ of coordinates and the start point of circle curve is $\boldsymbol{D}_{0}$.

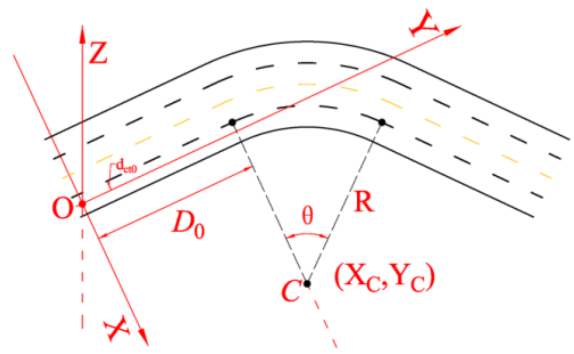

Fig.10 Coordinate system

The offset between vehicle and lane centerline is $d_{c t 0}$ at time $t_{0}$ (if lane centerline is located Y-axis left side the value is negative else it is positive.), the coordinate value of the centre of a circle is as follows,

$$
X_{C}=R+d_{c t 0}, Y_{C}=D_{0}
$$

The parameter models of lane centerline are as below,

$$
P(G)=\left\{\begin{aligned}
& x_{g}= d_{c t 0} \quad y_{g} \leq D_{0} \\
&\left\{\begin{array}{l}
y_{g}=R \sin \varphi_{g}+Y_{C} \\
x_{g}=
\end{array} \quad y_{g} \cos \varphi_{g}+X_{C}\right. \\
& y_{g}=\operatorname{ctg} \theta \cdot x_{g}+R \sin \theta+Y_{C}-\operatorname{ctg} \theta\left(-R \cos \theta+X_{C}\right) \\
& \quad\left(y_{g} \geq R \sin \theta+Y_{C}\right)
\end{aligned}\right.
$$

At any moment $t$, the space position coordinates of vehicle is $\left(x_{c t}, y_{c t}, z_{c t}\right)$, Among them $z_{c t}$ shows height of sighting line of the drivers, the speed is $v_{c t}$, the direction angle is $\varphi_{c t}$, (the angle between travel direction of the vehicle's center of mass and the direction of lane centerline), the lateral acceleration is $\alpha_{c t}$, the offset distance with lane centerline is $d_{c t}=\left|x_{c t}-d_{c t 0}\right|$, the ahead distance is $d_{c s t}$, the maximum offset distance is $d_{c t \max }$ between vehicle and lane centerline.

\section{Published by Atlantis Press




$$
-d_{\text {ctmax }} \leq d_{c t} \leq d_{\text {ctmax }}
$$

According to the desired trajectory feature above the six concave curves, their parameter models were to establish, as shown in Fig.11. Convex curve and take on opposition lane also can be reference to the modeling ideas of the six basic forms.

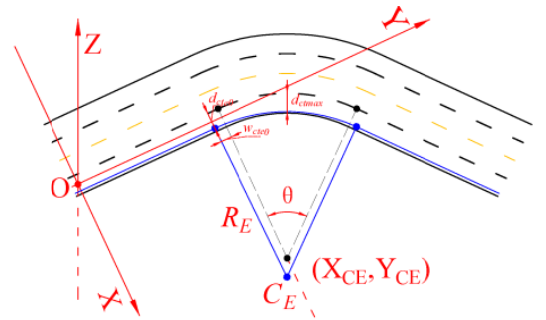

Fig.11 analysis of desired trajectory（a )

The symbols in Fig.11 are described as follows:

$R_{E} \longrightarrow$ radius of curve of desired trajectory;

$C_{E}$ center of the circle of desired trajectory;

$X_{C E}, Y_{C E}$ - ordinates of center of the circle of desired trajectory;

$d_{c t e 0}$ - - the lateral offset of desired trajectory starting point from the lane centerline

$w_{c t e 0}$ - the longtiudinal offset of desired trajectory starting point from the road strating poing.

It reaches the maximum lateral offset $d_{c t \max }$ that is the lateral offset of the desired trajectory midpoint from the lane centerline, steering angle keeps constant, thus here is the following equation,

$$
\begin{gathered}
R_{E}\left(1-\cos \frac{\theta}{2}\right)+d_{c t e 0}=R\left(1-\cos \frac{\theta}{2}\right)+d_{c t \max } \cdot \cos \frac{\theta}{2} \\
R_{E}=R+\left(d_{c t \max } \cdot \cos \frac{\theta}{2}-d_{c t e 0}\right) /\left(1-\cos \frac{\theta}{2}\right)
\end{gathered}
$$

Drivers desire curvature to reduce, therefore here is the following equation $R_{E} \geq R$, then,

$$
d_{c t e 0} \in\left[0, d_{c t \max } \cdot \cos \frac{\theta}{2}\right]
$$

When $d_{c t e 0}=0$, the starting point of desired trajectory is in lane centerline, the longitudinal offset between the starting point and the road starting point reaches the maximum, the curvature is the minimum.
When $d_{c t e 0}=d_{c t \max } \cdot \cos \frac{\theta}{2}$, the curvature keeps constant, the longitudinal distance between the starting point and the road starting point reaches the minimum that does not ensure the curvature to trend max.

$w_{c t e 0}$ is as follows,

$$
w_{c t e 0}=\left(d_{c t \max }-d_{c t e 0}\right) \sin \frac{\theta}{2} /\left(1-\cos \frac{\theta}{2}\right)
$$

Then,

$$
\begin{aligned}
& Y_{C E}=Y_{C}-w_{c t e 0} \\
& X_{C E}=X_{C}+\left(d_{c t \max }-d_{c t e 0}\right) \cos \frac{\theta}{2} /\left(1-\cos \frac{\theta}{2}\right)
\end{aligned}
$$

As a result, desired trajectory (a) parametric models including the staightway to join desired trajectory are as follows,

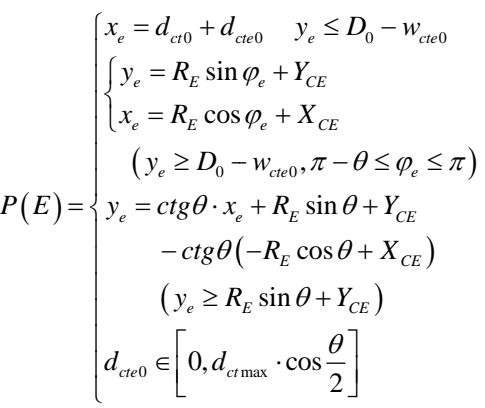

The other five desired trajectories models are similar to desired trajectory (a), there is not repeat them.

\subsection{Parameters analysis}

In order to study rationality of desired trajectory models, to change parameters in models except $\mathrm{R}$, $\theta, d_{c t \max }$ can to get the distribute figure, Shown in Fig.12, and steering angle is $50^{\circ}$.

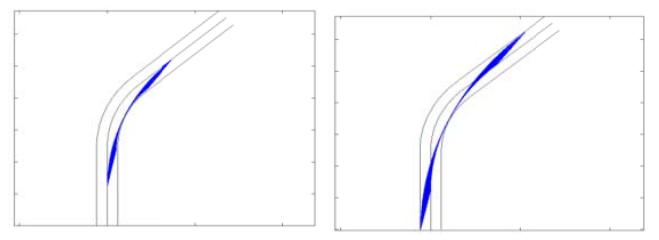

Desired trajectory (a) desired trajectory (b) 


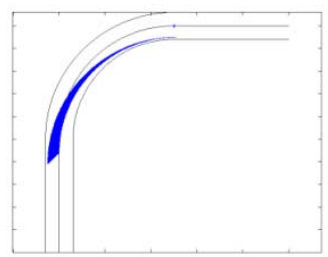

Desired trajectory (c)

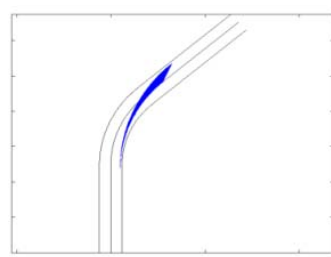

desired trajectory (d)

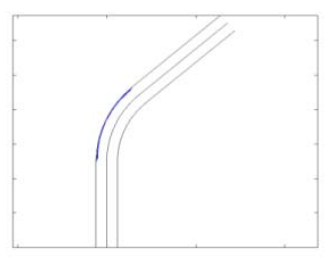

Desired trajectory (f)

Fig.12 Analysis of desired trajectory models

The longitudinal offset $w_{c t e o}$ indicates the forward trendency of the starting point of desired trajectory. Its models show that the longitudinal offset from the curve only related to the road curve, for example the desired trajectory (a), and steering angle is $40^{\circ} \sim 180^{\circ}$, regularity of the longitudinal offset $w_{c t e o}$ with the steering angle is shown Fig.13.

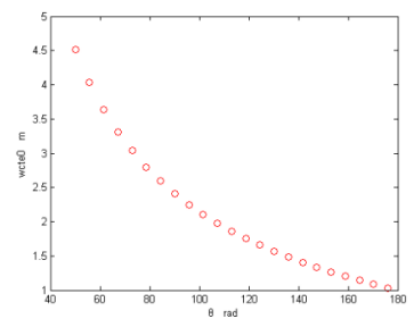

Fig.13 analysis of desired trajectory (a) $-w_{c t e 0}$

\section{Driver's desired speed}

The desired speed depends on three factors: the roadway geometrics, the characteristics of driver and the vehicle performance.

\subsection{The shaping mechanism of driver's desired speed}

Before the driver enters a horizontal curve, the vehicle travelling trajectory will be estimated, i.e. the desired trajectory according to the curve characteristics and road conditions through observation.Such driving experience can be achieved by a long term driving practice getting familiar with the vehicle and driving maneuvers, which is corresponding to the rules of cognitive psychology. For a certain vehicle, the relationships among turning radius, speed and the rotation range of the steering wheel is kept constant.

\subsection{Model of driver's desired speed}

Vehicle steering system varies under different speeds according to the typical vehicle engineering theoretics. At a low speed, the turning radius $R_{c}$ depends only on the front wheel rotation angle and the vehicle wheelbase $L_{c}$, but not the vehicle speed. At a high speed, side slip (or vehicle slip) may occur. The size of the slip angle is related to the tire lateral force ${ }^{8}$.

From analysing vehicle dynamic performance, the time for the steering system from instantaneous response to steady state is short, so the process of dynamic response can be ignored. Moreover, as most vehicles are designed as understeering, the relationships among $R_{c}, V_{c x}, \varphi_{c a}$ can be defined as formula (10). The turning angle of the front wheel is a ratio of the steering wheel angle to the vehicle transmission- ratio.

$$
\begin{gathered}
\varphi_{c a}=\varphi_{c s w} / i_{c} \\
R_{c}=\frac{L_{c}}{\varphi_{c s w} / i_{c}}\left[1+K_{c} V_{c x}{ }^{2}\right]
\end{gathered}
$$

Then the axial velocity of the vehicle mass center can be calculated by,

$$
\mathrm{V}_{c x}=\left[\frac{1}{K_{c}}\left(\frac{R_{c} \varphi_{c s w} / i_{c}-1}{L_{c}}\right)\right]^{\frac{1}{2}}
$$

Drivers always desire to travel with a desired trajectory, and the desired speed can be achieved by the $R_{e}$ instead of $R_{c}$ in Eq.12.

$$
\mathrm{V}_{E}=\left[\frac{1}{K_{c}}\left(\mathrm{R}_{E} \frac{\varphi_{c s w} / i_{c}}{L_{c}}-1\right)\right]^{\frac{1}{2}}
$$


The model indicates that the road geometric characteristics (curvature of horizontal curve), vehicle performance, and driver experiences will directly influence to the driver desired speed.

\subsection{Model Analysis}

A certain passenger car is taken as a case study of the model with the following parameters: $m=1818.2 \mathrm{~kg}$, $L=3.048 \mathrm{~m}, \quad a_{c}=1.463 \mathrm{~m}, \quad b_{c}=1.585 \mathrm{~m}, \quad k_{1}=62618 \mathrm{~N} / \mathrm{rad}$, $k_{2}=-110185 \mathrm{~N} / \mathrm{rad}, i_{c}=20$.

From Fig. 3 it is instructive to note that within a certain angle range of the steering wheel, desired speed increases with the growth of the steering wheel angle. It shows to complete the turning maneuver, the driver will choose a lower speed in this case, and a smaller angle of the front wheel will be needed. Otherwise a dangerous oversteer may occur. On the contrary, if the driver chooses a higher speed, s/he should also keep the angle of the front wheel in a larger value, or the vehicle may be said to have understeer.

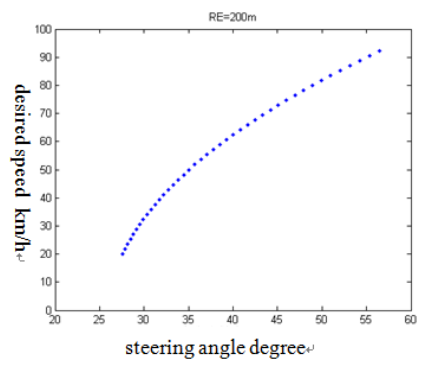

Fig.14 the relationship between steering wheel and desired speed of the study case

\section{Conclusions}

This paper discusses in depth driver expectation shaping mechanism by using a combination measurement of road geometer parameter, driver behavior and vehicle performance.

In our research, using various geometer parameters with different driving characteristics, the vehicle trajectory and speed were selected as key factors for describing completely the vehicle's operation condition. First of all, the generating process of the desired speed was described, and then the effect of driver's identification of vehicle dynamics on the desired speed was highlighted discussed by means of desired trajectory and control strategies. As a highlight work, the desired speed model that consisted of the desired trajectory, driver's experience and vehicle parameter has been developed, the numerical simulation results show the simulation-based model of driver expectation is used to driving behavior analysis in mountainous road.

\section{Acknowledgements}

This research was supported in part by the Programme of Introducing Talents of Discipline to Universities under grant B12022, National Nature Science Foundation of China under Grant 50878023, 51110305060, and the National Science \& Technology Pillar Program (2009BAG13A02).

\section{References}

1. Federal Highway Administration. Development of a Driver Vehicle Module for the Interactive Highway Safety Design Model. U S Department of transportation.2007.

2. Matthias Althoff, Olaf Stursberg, and Martin Buss. Safety assessment of driving behavior in multi-lane traffic for autonomous vehicles. Intelligent Vehicles Symposium, 2009 IEEE.

3. Guan Xin, Gao Zhenhai, Guo Konghui, Driver fuzzy decision model of vehicle preview course and simulation under typical road conditions, Automotive engineering. 23(1)(2001)13-17.(in Chinese)

4. GAO Zhen-hai, GUAN Xin, GUO Kong-hui, Driver fuzzy decision model of vehicle preview course, Natureal science journal of Jilin University of technology. 30(1)(2000)7-10. (in Chinese)

5. PAN Xiao-dong, DU Zhi-gang, JIANG Hong et al, Experiment research on relationship between the variation of drivers' heart rate and systolic blood pressure and a lignment of mountainous highway, Chinese Journal of Ergonomics. , 12(2) (2006) 16-18. (in Chinese)

6. Wuhong Wang, Xiaobei Jiang, Shuangcheng Xia, et al, Incident tree model and incident tree analysis method for quantified risk assessment: an in-depth accident study in traffic operation, Safety Science. 48(10) (2010) 1248-1262.

7. Wuhong Wang, Yan Mao, Jin Jing, et al, Driver's various information process and multi-ruled decision-making mechanism: a fundamental of intelligent driving shaping model, International Journal of Computational Intelligence Systems. 4(3) (2011) 297-305.

8. GUO Kong-hui, Vehicle handling stability,( Jilin People's Publishing House, Jilin,1983). (in Chinese) 